\title{
On the Solvability Complexity Index for Unbounded Selfadjoint and Schrödinger Operators
}

\author{
Frank Rösler(D)
}

\begin{abstract}
We study the solvability complexity index (SCI) for unbounded selfadjoint operators on separable Hilbert spaces and perturbations thereof. In particular, we show that if the extended essential spectrum of a selfadjoint operator is convex, then the SCI for computing its spectrum is equal to 1 . This result is then extended to relatively compact perturbations of such operators and applied to Schrödinger operators with (complex valued) potentials decaying at infinity to obtain SCI $=1$ in this case, as well.
\end{abstract}

Mathematics Subject Classification. Primary 35P99; Secondary 35Q40.

Keywords. Schrödinger operators, Spectral approximation, Computational complexity.

\section{Introduction}

The problem of computing spectra of partial differential operators is fundamental to many problems in physics with real world applications. Perhaps one of the most prominent examples of this is quantum mechanics, where the possible bound state energies of a particle subject to a force described by a potential function $V$ are given by the eigenvalues of the corresponding Schrödinger operator $-\Delta+V$. Generically, the spectral problem of such an operator cannot be solved explicitly and one has to resort to numerical methods. By practical constraints, any computer algorithm, which might be used to compute the spectrum, will only be able to handle a finite amount of information about the operator and perform a finite number of arithmetic operations on this information (in practice, this "finite amount of information" is usually given by some sort of discretisation of the domain, which approximates the infinite dimensional spectral problem by a finite dimensional one). In other words, any algorithm will always "ignore" an infinite 
amount of information about the operator. One might hope that by increasing the dimension of the approximation (or decreasing the step size of the discretisation), one will eventually obtain a reasonable approximation of the spectrum. Hence, it is a legitimate question to ask:

Given a class of operators $\Omega$, does there exist a sequence of algorithms $\Gamma_{n}$ such that $\Gamma_{n}(T) \rightarrow \sigma(T)$ (in an appropriate sense) for all $T \in \Omega$ ?

It turns out that the answer to the above question is not always in the affirmative. Indeed, it has been shown in [2] that if $\Omega=L(\mathcal{H})$ (the space of bounded operators on a separable Hilbert space $\mathcal{H}$ ), then for any sequence of algorithms there exists $T \in \Omega$ whose spectrum is not approximated by that sequence. This observation has led to the wider definition of the so-called Solvability Complexity Index (SCI), introduced in [9], of which we will now give a brief review.

Definition 1.1. A computational problem is a quadruple $(\Omega, \Lambda, \Xi, \mathcal{M})$, where

(i) $\Omega$ is a set, called the primary set,

(ii) $\Lambda$ is a set of complex valued functions on $\Omega$, called the evaluation set,

(iii) $\mathcal{M}$ is a metric space,

(iv) $\Xi: \Omega \rightarrow M$ is a map, called the problem function.

In the above definition, $\Omega$ is the set of objects that give rise to the computational problem, $\Lambda$ plays the role of providing the information accessible to the algorithm, and $\Xi: \Omega \rightarrow \mathcal{M}$ gives the quantity that one wishes to compute numerically.

An example of a computational problem in the sense of Definition 1.1 is given by the spectral problem discussed above. Indeed, given a separable Hilbert space $\mathcal{H}$ with orthonormal basis $\left\{e_{i}\right\}$, one can choose $\Omega=L(\mathcal{H}), \mathcal{M}=$ $\{$ compact subsets of $\mathbb{C}\}$, equipped with the Hausdorff metric, and $\Xi(T)=$ $\sigma(T)$. For the evaluation set one could choose $\Lambda:=\left\{f_{i j} \mid i, j \in \mathbb{N}\right\}$, where $f_{i j}(T)=\left\langle T e_{i}, e_{j}\right\rangle$ give the matrix elements of an operator with respect to the basis $\left\{e_{i}\right\}$.

Definition 1.2. Let $(\Omega, \Lambda, \Xi, \mathcal{M})$ be a computational problem. An arithmetic algorithm is a map $\Gamma: \Omega \rightarrow \mathcal{M}$ such that for each $T \in \Omega$ there exists a finite subset $\Lambda_{\Gamma}(T) \subset \Lambda$ such that

(i) the action of $\Gamma$ on $T$ depends only on $\{f(T)\}_{f \in \Lambda_{\Gamma}(T)}$,

(ii) for every $S \in \Omega$ with $f(T)=f(S)$ for all $f \in \Lambda_{\Gamma}(T)$ one has $\Lambda_{\Gamma}(S)=$ $\Lambda_{\Gamma}(T)$

(iii) the action of $\Gamma$ on $T$ consists of performing only finitely many arithmetic operations on $\{f(T)\}_{f \in \Lambda_{\Gamma}(T)}$.

We will refer to any arithmetic algorithm simply as an algorithm from now on. For more general concepts the reader may consult [2].

In [2] it has been shown that if $\Omega$ is the set of compact operators on a separable Hilbert space $\mathcal{H}$, then there exists a sequence of algorithms $\Gamma_{n}$ : $\Omega \rightarrow \mathbb{C}$ such that $\Gamma_{n}(T) \rightarrow \sigma(T)$ (in Hausdorff sense) for all $T \in \Omega$, while for 
the set of bounded selfadjoint operators $\Omega=\left\{T \in L(\mathcal{H}) \mid T^{*}=T\right\}$ this is not possible.

However, it turns out that there exists a family $\Gamma_{m n}$ of algorithms such that

$$
\lim _{n \rightarrow \infty} \lim _{m \rightarrow \infty} \Gamma_{m n}(T)=\sigma(T)
$$

for all bounded selfadjoint operators. Hence, it is possible to compute the spectrum of non-compact operators using algorithms, but the number of limits required may increase (this general phenomenon has first been observed by Doyle and McMullen in the context of finding zeros of polynomials, cf. [7]). In order to capture this phenomenon, the following definition has been made

Definition 1.3. [2] Let $(\Omega, \Lambda, \Xi, \mathcal{M})$ be a computational problem. A tower of algorithms of height $k$ is a family $\Gamma_{n_{1}, n_{2}, \ldots, n_{k}}: \Omega \rightarrow \mathcal{M}$ of arithmetic algorithms such that for all $T \in \Omega$

$$
\Xi(T)=\lim _{n_{k} \rightarrow \infty} \cdots \lim _{n_{1} \rightarrow \infty} \Gamma_{n_{1}, n_{2}, \ldots, n_{k}}(T) .
$$

The examples above show that the number of limits required to compute the problem function $\Xi$ is a measure for the numerical complexity of the underlying computational problem. This motivates the

Definition 1.4. [2] A computational problem $(\Omega, \Lambda, \Xi, \mathcal{M})$ is said to have $S o l v$ ability Complexity Index $k$ if $k$ is the smallest integer for which there exists a tower of algorithms of height $k$ that computes $\Xi$.

If a computational problem has solvability complexity index $k$, we write $\operatorname{SCI}(\Omega, \Lambda, \Xi, \mathcal{M})=k$.

Remark 1.5. In this article we are mainly interested in the spectral problem and will therefore write $\operatorname{SCI}(\Omega, \Lambda)$ instead of $\operatorname{SCI}(\Omega, \Lambda, \Xi, \mathcal{M})$, where it is understood that $\Xi(T)=\sigma(T)$ and $\mathcal{M}$ is the set of closed subsets of $\mathbb{C}$ equipped with the Attouch-Wets metric $d_{\mathrm{AW}}$ defined as

$$
d_{\mathrm{AW}}(A, B)=\sum_{i=1}^{\infty} 2^{-i} \min \left\{1, \sup _{|x|<i}|\operatorname{dist}(x, A)-\operatorname{dist}(x, B)|\right\} .
$$

(Note that if $A, B \subset \mathbb{C}$ are bounded, then $d_{\mathrm{AW}}$ coincides with the Hausdorff distance.)

In practice it is often important to have control of the approximation error $d\left(\Gamma_{n_{1}, \ldots, n_{k}}(T), \Xi(T)\right)$ for all $T \in \Omega$. It is straightforward to show, however, that such an estimate is impossible to obtain as soon as $\operatorname{SCI}(\Omega, \Lambda, \Xi, \mathcal{M})>1$ (cf. [2, Thm. 6.1]). Indeed, it is easy to see that if for a tower of algorithms $\Gamma_{n_{1}, \ldots, n_{k}}$ there exist subsequences $n_{1}(m), \ldots, n_{k}(m)$ such that $\Gamma_{n_{1}(m), \ldots, n_{k}(m)}$ $(T) \rightarrow 0$ for all $T \in \Omega$, then $\tilde{\Gamma}_{m}:=\Gamma_{n_{1}(m), \ldots, n_{k}(m)}$ is in fact a tower of height 1 for $\Omega$ and hence $\operatorname{SCI}(\Omega)=1$.

For this reason, it is of particular interest to find classes $\Omega$ of operators for which $\operatorname{SCI}(\Omega, \Lambda, \sigma(\cdot))=1$ (with appropriately chosen $\Lambda$ ). The present 
article addresses precisely this question. In fact, we will show that for selfadjoint operators whose extended essential spectrum (see (2.2)) is convex, we have $\mathrm{SCI}=1$. This is done by explicitly constructing a sequence of arithmetic algorithms which computes the spectrum of any such operator. The result is then extended to certain relatively compact perturbations of such operators. We stress that the new aspect of our work is to consider the shape of the essential spectrum as a relevant criterion for reducing the numerical complexity of the spectral problem. As an application of this approach, we will show that our results apply to non-selfadjoint Schrödinger operators with certain well behaved potentials.

The problem of determining the SCI for spectral problems has previously been studied in [2,9] for operator in abstract Hilbert spaces, as well as for partial differential operators. Previous results include

Bounded operators Let $\mathcal{H}, \Lambda$ be as in the example above Definition 1.2. It was shown in $[2$, Th. 3.3 , Th. 3.7] that then

$$
\begin{array}{ll}
\operatorname{SCI}(\Omega, \sigma(\cdot))=3 & \text { if } \Omega=L(\mathcal{H}) \\
\operatorname{SCI}(\Omega, \sigma(\cdot))=2 & \text { if } \Omega=\{T \in L(\mathcal{H}) \mid T \text { selfadjoint }\} \\
\operatorname{SCI}(\Omega, \sigma(\cdot))=1 & \text { if } \Omega=\mathcal{K}(\mathcal{H}),
\end{array}
$$

where $\mathcal{K}(\mathcal{H})$ denotes the set of compact operators. The last of the above bounds, $\operatorname{SCI}(\mathcal{K}(\mathcal{H}), \sigma(\cdot))=1$, is related to the fact that compact operators can be approximated in operator norm by finite range operators.

Schrödinger operators In [2], the SCI for the spectral problem of Schrödinger operators with complex valued potentials $V$ has been studied. It has been shown that if

$$
\Omega=\{-\Delta+V \mid V \text { is sectorial and }|V(x)| \rightarrow \infty \text { as }|x| \rightarrow \infty\},
$$

then $\operatorname{SCI}(\Omega, \sigma(\cdot))=1$. The proof relies on the fact that operators as in (1.1) have compact resolvent.

In the case of bounded potentials, one lacks compact resolvent and the situation is somewhat more difficult. It has been shown in [2, Th. 4.2] that if $\Omega$ denotes the set of Schrödinegr operators on $\mathbb{R}^{d}$ with $V$ bounded and of bounded variation, then $\operatorname{SCI}(\Omega, \sigma(\cdot)) \leq 2$. It has since then been an open problem, whether without any additional information the SCI of this problem is equal to one or two.

The SCI of certain unbounded operators in separable Hilbert spaces, whose matrix representation is banded, has been studied in [9].

In this article, we will take a step towards closing this gap. We will prove that if $M>0$ and $\Omega$ denotes the set of all Schrödinger operators $-\Delta+V$ with $\operatorname{supp}(V) \subset B_{M}(0)$ and $|\nabla V| \leq M$, then $\operatorname{SCI}(\Omega, \sigma(\cdot))=1$ (for the precise statement, see Sect. 4$)$. This is done by first proving two abstract theorems about the SCI of selfadjoint operators which are of independent interest. The proofs of these abstract results rely on recent developments in the theory of essential numerical ranges for unbounded operators, cf. [3]. The main theorems of this article are Theorems 2.1, 3.1 and 4.3. 
The question as to wether the assumption on the decay of $V$ is essential for having SCI = 1 remains an interesting open problem and will be addressed in future work.

\section{Selfadjoint Operators}

Let $\mathcal{H}$ be a separable Hilbert space and $\mathcal{H}_{n} \subset \mathcal{H}$ be a sequence of finite dimensional subspaces such that $\mathcal{H}_{n} \subset \mathcal{H}_{n+1}$ for all $n \in \mathbb{N}$ and $P_{n} \stackrel{s}{\rightarrow} I$, where $P_{n}$ denotes the orthogonal projection onto $\mathcal{H}_{n}$. Define

$$
\Omega_{1}:=\left\{\begin{array}{l|l}
T: \operatorname{dom}(T) \rightarrow \mathcal{H} & \begin{array}{l}
T \text { selfadjoint, } \widehat{\sigma}_{e}(T) \text { convex } \\
\text { and } \bigcup_{n \in \mathbb{N}} \mathcal{H}_{n} \text { is a core of } T
\end{array}
\end{array}\right\},
$$

where

$$
\widehat{\sigma}_{e}(T)=\sigma_{e 2}(T) \cup \begin{cases}\{+\infty\}, & \text { if } T \text { unbounded above } \\ \{-\infty\}, & \text { if } T \text { unbounded below }\end{cases}
$$

and

$$
\sigma_{e 2}(T)=\left\{\lambda \in \mathbb{C} \mid \exists\left(x_{k}\right) \subset \operatorname{dom}(T):\left\|x_{k}\right\|=1 \forall k, x_{k} \rightarrow 0,\left\|(T-\lambda) x_{k}\right\| \rightarrow 0\right\} .
$$

Furthermore, for each $n \in \mathbb{N}$, let $\left\{e_{1}^{(n)}, \ldots, e_{k_{n}}^{(n)}\right\}$ be an orthonormal basis of $\mathcal{H}_{n}$ and define

$$
\Lambda_{1}:=\left\{f_{i, j, n} \mid 1 \leq i, j \leq k_{n}, n \in \mathbb{N}\right\}
$$

where $f_{i, j, n}: T \mapsto\left\langle T e_{i}^{(n)}, e_{j}^{(n)}\right\rangle$ are the evaluation functions producing the $(i, j)$ th matrix elements. This is the set of information accessible to the algorithm.

Theorem 2.1. We have $\operatorname{SCI}\left(\Omega_{1}, \Lambda_{1}, \sigma(\cdot)\right)=1$.

Remark 2.2. (i) Note that Theorem 2.1 in particular applies to bounded selfadjoint operators with convex essential spectrum. In this sense, Theorem 2.1 can be viewed as an extension of [2, Th. 3.7], where it was shown that SCI $=1$ for the set of all compact operators (which naturally satisfy $\left.\sigma_{e}(T) \subset\{0\}\right)$.

(ii) Theorem 2.1 is optimal in the sense that the selfadjointness assumption in (2.1) cannot be dropped. Indeed, counterexamples show that SCI $\geq 2$ for non selfadjoint bounded operators with convex essential spectrum (cf. [2, Proof of Th. 3.7, Step II] for an explicit construction).

Remark 2.3. In addition to (2.3) we will need another version of the essential spectrum, which is sometimes denoted $\sigma_{e 5}$. Let $H$ be a closed, densely defined operator on $\mathcal{H}$. Then

$$
\sigma_{e 5}(H):=\mathbb{C} \backslash \Delta_{5}(H),
$$

where $\Delta_{5}(H)$ denotes the union of all components of the set $\{\lambda \in \mathbb{C} \mid H-$ $\lambda$ is semi-Fredholm $\}$ which intersect $\rho(H)$. Note that the definitions $(2.3)$ and (2.5) do not agree in general. However, it can be shown that for selfadjoint 
operators $T$ on $\mathcal{H}$, one always has $\sigma_{e 2}(T)=\sigma_{e 5}(T)$ (cf. [8, Th. IX.1.6]). For this reason, we will simply use the notation $\sigma_{e}(T)$ to denote the essential spectrum, whenever the operators in question are selfadjoint.

\subsection{Definition of the Algorithm}

Let $T \in \Omega_{1}$ and define the truncated operator

$$
T_{n}:=\left.P_{n} T\right|_{\mathcal{H}_{n}} .
$$

This operator can be represented by a finite dimensional (square) matrix with elements $\left(T_{n}\right)_{i j}=\left\langle T e_{i}^{(n)}, e_{j}^{(n)}\right\rangle$. Moreover, let $G_{n}^{\mathbb{R}}:=\frac{1}{n} \mathbb{Z} \cap(-n, n) \subset \mathbb{R}$.

Lemma 2.4. Let $\lambda \in G_{n}^{\mathbb{R}}$ and denote by $s(\cdot)$ the smallest singular value of $a$ matrix. Then

(i) For all $n$ and $\lambda$, we have $s\left(T_{n}-\lambda\right)=\left\|\left(T_{n}-\lambda\right)^{-1}\right\|_{L\left(\mathcal{H}_{n}\right)}^{-1}$.

(ii) For any $q>0$, testing whether $s\left(T_{n}-\lambda\right)>q$ requires only finitely many arithmetic operations on the matrix elements of $T_{n}$.

with the convention that $\left\|\left(T_{n}-\lambda\right)^{-1}\right\|^{-1}=0$ for $\lambda \in \sigma\left(T_{n}\right)$.

Proof. Part (i) was proved in [9], while part (ii) follows by noting that $s\left(T_{n}-\right.$ $\lambda)>q$ is equivalent to $\left(T_{n}-\lambda\right)^{*}\left(T_{n}-\lambda\right)-q^{2} I$ being positive definite; see $[2$, Prop. 10.1] for a full proof.

For $n \in \mathbb{N}$ we define a map $\Gamma_{n}^{(1)}: \Omega_{1} \rightarrow\{$ closed subsets of $\mathbb{C}\}$ by

$$
\Gamma_{n}^{(1)}(T):=\left\{\lambda \in G_{n}^{\mathbb{R}} \mid s\left(T_{n}-\lambda\right) \leq \frac{1}{n}\right\} .
$$

Then, by the above lemma, each $\Gamma_{n}^{(1)}$ is an arithmetic tower of height one in the sense of Definition 1.3. Clearly, $\Gamma_{n}^{(1)}(T) \subset \sigma_{\frac{1}{n}}\left(T_{n}\right)$ for all $n$, where $\sigma_{\frac{1}{n}}(\cdot)$ denotes the $\frac{1}{n}$-pseudospectrum, i.e. $\sigma_{\frac{1}{n}}\left(T_{n}\right)=\left\{z \in \mathbb{C} \mid\left\|\left(T_{n}-z\right)^{-1}\right\|>n\right\}$. Next we prove a version of the second resolvent identity for our operator approximation.

Lemma 2.5. Let $T: \operatorname{dom}(T) \rightarrow \mathcal{H}$ be selfadjoint, $\bigcup_{n} \mathcal{H}_{n}$ form a core of $T$ and $T_{n}$ be defined as in (2.6). Then each $T_{n}$ is selfadjoint on $\mathcal{H}_{n}$ and $T_{n} \rightarrow T$ in strong resolvent sense.

Proof. We start by showing that each $T_{n}$ is selfadjoint. First note that each $T_{n}$ is automatically bounded, since the $\mathcal{H}_{n}$ are finite dimensional. Now let $x, y \in \mathcal{H}_{n}$. Then we have

$$
\begin{aligned}
\left\langle T_{n} x, y\right\rangle & =\left\langle P_{n} T x, y\right\rangle=\left\langle T x, P_{n} y\right\rangle=\langle T x, y\rangle \\
& =\langle x, T y\rangle=\left\langle P_{n} x, T y\right\rangle=\left\langle x, P_{n} T y\right\rangle=\left\langle x, T_{n} y\right\rangle .
\end{aligned}
$$

and hence $T_{n}$ is selfadjoint. The claim now follows directly from [13, Satz 9.29], since $\bigcup_{n} \mathcal{H}_{n}$ is a core for $T$ and $P_{n}$ converges strongly to the identity. 


\subsection{General Results on Spectral and Pseudospectral Pollution}

In this subsection we collect facts about spectral and pseudospectral pollution for closed, densely defined operators $H, H_{n}$ on $\mathcal{H}$, which are not necessarily selfadjoint. These results will be used in later sections. The sets of spectral and pseudospectral pollution are defined, respectively, as

$$
\begin{aligned}
\sigma_{\text {poll }}(H) & =\left\{z \in \mathbb{C} \backslash \sigma(H) \mid \exists \text { sequence } \lambda_{n} \in \sigma\left(H_{n}\right): \lambda_{n} \rightarrow \lambda\right\} \\
\sigma_{\varepsilon, \text { poll }}(H) & =\left\{z \in \mathbb{C} \backslash \sigma_{\varepsilon}(H) \mid \exists \text { sequence } \lambda_{n} \in \sigma_{\varepsilon}\left(H_{n}\right): \lambda_{n} \rightarrow \lambda\right\} .
\end{aligned}
$$

The following definitions from [3], which are related to the essential spectrum, will be used frequently in the sequel. The limiting essential spectrum:

$$
\sigma_{e}\left(\left(H_{n}\right)_{n \in \mathbb{N}}\right):=\left\{\begin{array}{l|l}
\lambda \in \mathbb{C} & \begin{array}{l}
\exists x_{k} \in \operatorname{dom}\left(H_{n_{k}}\right) \text { s.t. } \\
\left\|x_{k}\right\|=1 \forall k, x_{k} \rightarrow 0,\left\|\left(H_{n_{k}}-\lambda\right) x_{k}\right\| \rightarrow 0
\end{array}
\end{array}\right\},
$$

the limiting $\varepsilon$-near spectrum:

$$
\Lambda_{e, \varepsilon}\left(\left(H_{n}\right)_{n \in \mathbb{N}}\right):=\left\{\begin{array}{ll}
\lambda \in \mathbb{C} \mid \begin{array}{l}
\exists x_{k} \in \operatorname{dom}\left(H_{n_{k}}\right) \text { s.t. } \\
\left\|x_{k}\right\|=1 \forall k, x_{k} \rightarrow 0,\left\|\left(H_{n_{k}}-\lambda\right) x_{k}\right\| \rightarrow \varepsilon
\end{array}
\end{array}\right\},
$$

the essential numerical range

$W_{e}(H):=\left\{\lambda \in \mathbb{C} \mid \exists x_{k} \in \operatorname{dom}(H)\right.$ s.t. $\left.\left\|x_{k}\right\|=1 \forall k, x_{k} \rightarrow 0,\left\langle H x_{k}, x_{k}\right\rangle \rightarrow \lambda\right\}$ and the limiting essential numerical range

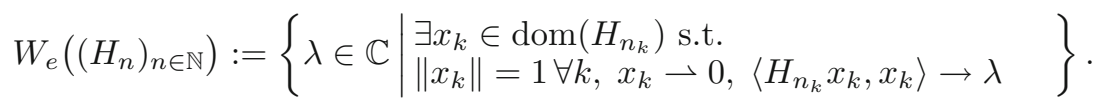

The essential limiting spectrum was originally introduced in [1] in the context of Galerkin approximation and later adapted to a more general framework in $[4,5]$, where the set $\Lambda_{e, \varepsilon}\left(\left(H_{n}\right)_{n \in \mathbb{N}}\right)$ was introduced. The essential numerical range was originally introduced by Stampfli and Williams in [12] for bounded operators and recently extended to unbounded operators in [3]. It was shown there that the essential numerical range is a convenient tool when studying spectral and pseudospectral pollution of operator approximations. This fact will prove useful to our purpose as we shall see in the following.

In order to prove the next lemma, we need a fact about closures of pseudospectra.

Lemma 2.6. For all operators $H$ on $\mathcal{H}$ of the form $H=T+V$, where $T$ is selfadjoint and $V$ is bounded, and all $\varepsilon<\varepsilon^{\prime}$ one has

$$
\overline{\sigma_{\varepsilon}(H)} \subset \sigma_{\varepsilon^{\prime}}(H) .
$$

Proof. This follows from the fact that the resolvent norm of any such operator tends to 0 at i $\infty$ and hence cannot be constant on an open set (cf. [6, Th. $3.2])$. Indeed, in this case we have

$$
\overline{\sigma_{\varepsilon}(H)}=\left\{z \mid\left\|(H-z)^{-1}\right\| \geq \varepsilon^{-1}\right\} \subset\left\{z \mid\left\|(H-z)^{-1}\right\|>\varepsilon^{\prime-1}\right\}=\sigma_{\varepsilon^{\prime}}(H) .
$$

Lemma 2.7. (i) For any closed, densely defined operator $H$ on $\mathcal{H}$ one has

$$
\bigcap_{\varepsilon>0} \bigcup_{\delta \in(0, \varepsilon]} \Lambda_{e, \delta}\left(\left(H_{n}\right)_{n \in \mathbb{N}}\right) \subset \sigma_{e}\left(\left(H_{n}\right)_{n \in \mathbb{N}}\right) \text {. }
$$


(ii) The above inclusion holds, if $\bigcap_{\varepsilon>0} \bigcup_{\delta \in(0, \varepsilon]}$ is replaced by $\bigcap_{k} \bigcup_{\delta \in\left(0, \varepsilon_{k}\right]}$ for any sequence $\left(\varepsilon_{k}\right)$ with $\varepsilon_{k} \rightarrow 0$.

Proof. We first prove (i). Let $\lambda \in \bigcap_{\varepsilon>0} \bigcup_{\delta \in(0, \varepsilon]} \Lambda_{e, \delta}\left(\left(H_{n}\right)_{n \in \mathbb{N}}\right)$. Then for all $\varepsilon>0$ there exists $\delta \in(0, \varepsilon]$ and a sequence $\left(x_{k}\right)$ with $x_{k} \in \operatorname{dom}\left(H_{n_{k}}\right)$ (for some subsequence $\left.\left(n_{k}\right)\right)$ such that

- $\left\|x_{k}\right\|=1$ for all $k$

- $x_{k} \rightarrow 0$ as $k \rightarrow \infty$

- $\left\|\left(H_{n_{k}}-\lambda\right) x_{k}\right\| \rightarrow \delta$.

Hence, for every $m \in \mathbb{N}$ there exists a sequence $\left(x_{k}^{(m)}\right)_{k \in \mathbb{N}}$ with $\left\|x_{k}^{(m)}\right\|=1$,

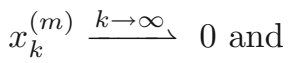

$$
\lim _{k \rightarrow \infty}\left\|\left(H_{n_{k}(m)}-\lambda\right) x_{k}^{(m)}\right\|<\frac{1}{m} .
$$

The notation $n_{k}(m)$ indicates that the corresponding subsequence of $\left(H_{n}\right)$ depends on $m$. Now, construct a diagonal sequence as follows. Since $\mathcal{H}$ is separable, the weak topology is metrisable on the unit ball. Let $d$ denote a corresponding metric. Now, for any given $m \in \mathbb{N}$, choose $k_{m} \in \mathbb{N}$ large enough such that

$$
\begin{aligned}
d\left(x_{k_{m}}^{(m)}, 0\right) & <\frac{1}{m} \\
\left\|\left(H_{n_{k_{m}}(m)}-\lambda\right) x_{k_{m}}^{(m)}\right\| & <\frac{1}{m} .
\end{aligned}
$$

Then for the sequence $y_{m}:=x_{k_{m}}^{(m)}$, one has $\left\|y_{m}\right\|=1$ for all $m, d(y, 0) \rightarrow 0$ and $\left\|\left(H_{n_{k_{m}}(m)}-\lambda\right) y_{m}\right\| \rightarrow 0$ as $m \rightarrow \infty$. Hence $\lambda \in \sigma_{e}\left(\left(H_{n}\right)_{n \in \mathbb{N}}\right)$.

The proof of claim (ii) is now immediate, because the sequence of sets $\bigcup_{\delta \in(0, \varepsilon]} \Lambda_{e, \delta}\left(\left(H_{n}\right)_{n \in \mathbb{N}}\right)$ is shrinking with $\varepsilon$.

Finally, we prove the following characterisation of convergence of sets in the Attouch-Wets metric. We recall that $d_{\mathrm{AW}}\left(X_{n}, X\right) \rightarrow 0$ if and only if $d_{K}\left(X_{n}, X\right) \rightarrow 0$ for all $K \subset \mathbb{C}$ compact, where

$$
d_{K}(X, Y):=\max \left\{\sup _{x \in X \cap K} \operatorname{dist}(x, Y), \sup _{y \in Y \cap K} \operatorname{dist}(y, X)\right\} .
$$

Proposition 2.8. Let $X, X_{n}, n \in \mathbb{N}$ be closed subsets of $\mathbb{C}$. Assume that

(a) If $\lambda_{n} \in X_{n}$ and $\lambda_{n} \rightarrow \lambda$, then $\lambda \in X$.

(b) If $\lambda \in X$, then there exist $\lambda_{n} \in X_{n}$ with $\lambda_{n} \rightarrow \lambda$.

Then one has $d_{\mathrm{AW}}\left(X_{n}, X\right) \rightarrow 0$.

Proof. Let $K \subset \mathbb{C}$ be compact. We will show that if (a), (b) hold, then both distances $\sup _{z \in X_{n} \cap K} \operatorname{dist}(z, X)$ and $\sup _{w \in X \cap K} \operatorname{dist}\left(w, X_{n}\right)$ converge to zero. We begin with the latter.

Let $\varepsilon>0$. For all $w \in X \cap K$, the ball $B_{\varepsilon}(w)$ contains infinitely many elements $z_{n} \in X_{n}$, by (b). The collection $\left\{B_{\varepsilon}(w) \mid w \in X \cap K\right\}$ forms an open cover of the compact set $X \cap K$. Hence, there exist finitely many $w_{1}, \ldots, w_{k} \in$ $X \cap K$ such that $B_{\varepsilon}\left(w_{1}\right), \ldots, B_{\varepsilon}\left(w_{k}\right)$ cover $X \cap K$. Now, any $w \in X \cap K$ 
is contained in some $B_{\varepsilon}\left(w_{i}\right)$ and hence $\operatorname{dist}\left(w, X_{n}\right)<\varepsilon$ for any $w \in X \cap$ $K$, as soon as $n=n(i)$ is large enough. But since there are only finitely many $B_{\varepsilon}\left(w_{i}\right)$, one will have $\operatorname{dist}\left(w, X_{n_{0}}\right)<2 \varepsilon$ for all $w \in X \cap K$ for $n_{0}=$ $\max \left\{n_{i} \mid i=1, \ldots, k\right\}$.

To show that $\sup _{z \in X_{n} \cap K} \operatorname{dist}(z, X) \rightarrow 0$ as $n \rightarrow \infty$, note that since all sets $X_{n} \cap K$ are compact, we can choose a sequence $z_{n} \in X_{n} \cap K$ such that

$$
\sup _{z \in X_{n} \cap K} \operatorname{dist}(z, X)=\operatorname{dist}\left(z_{n}, X\right) .
$$

Since the sequence $\left(z_{n}\right)$ is obviously bounded, we can extract a convergent subsequence $z_{n_{j}} \rightarrow z_{0} \in K$. Now use assertion (a) from above to conclude that in fact $z_{0} \in X \cap K$. This readily implies

$$
\sup _{z \in X_{n_{j}} \cap K} \operatorname{dist}(z, X)=\operatorname{dist}\left(z_{n_{j}}, X\right) \rightarrow 0 .
$$

Since the same reasoning can be applied to every subsequence of the sequence

$$
\left(\sup _{z \in X_{n} \cap K} \operatorname{dist}(z, X)\right)_{n \in \mathbb{N}},
$$

we conclude that the whole sequence converges to zero.

\subsection{Proof of Theorem 2.1}

Next, we prove convergence of the algorithm $\Gamma_{n}^{(1)}$. By the conditions in (2.1) and Lemma 2.5, we have $T_{n} \rightarrow T$ in strong resolvent sense for all $T \in \Omega$.

Lemma 2.9. If $\lambda_{n} \in \Gamma_{n}^{(1)}(T), n \in \mathbb{N}$ and $\lambda_{n} \rightarrow \lambda$, then

$$
\lambda \in \sigma(T) \cup \sigma_{e}\left(\left(T_{n}\right)_{n \in \mathbb{N}}\right) .
$$

Proof. By definition of $\Gamma_{n}^{(1)}$, one has that

$$
\frac{1}{n} \geq\left\|\left(\lambda_{n}-T_{n}\right)^{-1}\right\|^{-1} \geq \operatorname{dist}\left(\lambda_{n}, \sigma\left(T_{n}\right)\right)
$$

for all $n \in \mathbb{N}$. Hence, there exists a sequence $z_{n} \in \sigma\left(T_{n}\right)$ such that $\mid z_{n}-$ $\lambda_{n} \mid \rightarrow 0$ and consequently $z_{n} \rightarrow \lambda$. We conclude from [5, Th. 2.3] that $\lambda \in \sigma(T) \cup \sigma_{e}\left(\left(T_{n}\right)_{n \in \mathbb{N}}\right)$.

To conclude, we apply [3, Th. 6.1] to show that spectral pollution is in fact absent for $T \in \Omega_{1}$. Indeed, let $\lambda_{n} \in \Gamma_{n}^{(1)}(T)$ with $\lambda_{n} \rightarrow \lambda$. Then by Lemma 2.9 and [3, Prop. 5.6, Th. 6.1], we get

$$
\begin{aligned}
\lambda & \in \sigma(T) \cup \sigma_{e}\left(\left(T_{n}\right)_{n \in \mathbb{N}}\right) \\
& \subset \sigma(T) \cup W_{e}(T) \\
& =\sigma(T) \cup \operatorname{conv}\left(\widehat{\sigma}_{e}(T)\right) \backslash\{ \pm \infty\} \\
& =\sigma(T) \cup \sigma_{e}(T) \\
& =\sigma(T) .
\end{aligned}
$$

It remains to prove spectral inclusion, i.e. nothing is missed by $\Gamma_{n}^{(1)}(T)$.

Lemma 2.10. For every $\lambda \in \sigma(T)$ there exist $\lambda_{n} \in \Gamma_{n}^{(1)}(T)$ such that $\lambda_{n} \rightarrow \lambda$. 
Proof. Let $\lambda \in \sigma(T)$. A simple adaption of the proof of [11, Th. VIII.24] shows that there exists a sequence $\left(\mu_{n}\right)$ with $\mu_{n} \in \sigma\left(T_{n}\right)$ and $\mu_{n} \rightarrow \lambda$.

For each $n$, there exists $\lambda_{n} \in G_{n}^{\mathbb{R}}$ such that $\left|\mu_{n}-\lambda_{n}\right|<\frac{1}{n}$ and hence $\left\|\left(T_{n}-\lambda_{n}\right)^{-1}\right\|_{L\left(\mathcal{H}_{n}\right)} \geq n$ which implies $\lambda_{n} \in \Gamma_{n}^{(1)}(T)$. Since $\left|\mu_{n}-\lambda_{n}\right| \rightarrow 0$ and $\mu_{n} \rightarrow \lambda$, it follows that $\lambda_{n} \rightarrow \lambda$.

Conclusion We have shown that

(a) If $\lambda_{n} \in \Gamma_{n}^{(1)}(T)$ and $\lambda_{n} \rightarrow \lambda$, then $\lambda \in \sigma(T)$.

(b) If $\lambda \in \sigma(T)$, then there exist $\lambda_{n} \in \Gamma_{n}^{(1)}(T)$ with $\lambda_{n} \rightarrow \lambda$.

By Proposition 2.8, this implies Attouch-Wets convergence.

\section{Relatively Compact Perturbations}

In this section we show that Theorem 2.1 remains true for certain relatively compact, bounded perturbations of selfadjoint operators. More precisely, we have

Theorem 3.1. Define a computational problem by

$$
\Omega_{2}:=\left\{\begin{array}{l|l}
H=T+V: \operatorname{dom}(T) \rightarrow \mathcal{H} & \begin{array}{l}
T \text { selfadjoint, semibounded, } \\
\bigcup_{n \in \mathbb{N}} \mathcal{H}_{n} \text { core for } T, \\
\sigma(T)=\sigma_{e}(T), \widehat{\sigma}_{e}(T) \text { convex, } \\
V \in L(\mathcal{H}) \text { and } V, V^{*} \text { are } T \text {-compact. }
\end{array}
\end{array}\right\}
$$

For every $H \in \Omega_{2}$, choose a decomposition $H=T+V$ as in the definition of $\Omega_{2}$ and define the maps $s_{T}(H):=T$ and $s_{V}(H):=V$. Then let

$$
\Lambda_{2}:=\left\{f_{i, j, n} \circ s_{T} \mid 1 \leq i, j \leq n, n \in \mathbb{N}\right\} \cup\left\{f_{i, j, n} \circ s_{V} \mid 1 \leq i, j \leq n, n \in \mathbb{N}\right\},
$$

where $f_{i, j, n}$ are the evaluation functions producing the $(i, j)$ th matrix elements (see (2.4)). Then one has $\operatorname{SCI}\left(\Omega_{2}, \Lambda_{2}, \sigma(\cdot)\right)=1$.

Remark 3.2. (i) Note that the information provided to the algorithm in $\Lambda_{2}$ includes the decomposition of $H \in \Omega_{2}$ into a selfadjoint part $T$ and a perturbation $V$. This means, that the algorithm does not have to compute this decomposition. It gets it for free. This is a reasonable assumption in many applications as we will see in Sect. 4 .

(ii) In fact, the assumptions in the definition of $\Omega_{2}$ imply that $\sigma(T)$ is convex. Indeed, for any selfadjoint operator with purely essential spectrum, $\widehat{\sigma}_{e}(T)$ is convex if and only if $\sigma(T)$ is convex.

Note the additional assumption $\sigma(T)=\sigma_{e}(T)$ in the selfadjoint part $T$. This will be needed later in order to exclude spectral pollution of the algorithm.

\subsection{Proof of Theorem 3.1}

Spectrum of $\boldsymbol{H}$. The proof of Theorem 3.1 is via perturbation theory. We first focus on the spectrum of an operator $H \in \Omega_{2}$. Recall the definitions of the essential spectra $\sigma_{e 2}, \sigma_{e 5}$ from Sect. 2. In the proof, we will need the following results, which are classical. 
Theorem 3.3. [8, Th. IX.1.5] For any closed, densely defined operator $H$ on $\mathcal{H}$, one has $\lambda \notin \sigma_{e 5}(H)$ if and only if $H-\lambda$ is Fredholm with $\operatorname{ind}(H-\lambda)=0$ and a deleted neighbourhood of $\lambda$ lies in $\rho(H)$.

In other words, if $\lambda \notin \sigma_{e 5}(H)$, then $\lambda$ is an isolated eigenvalue of finite multiplicity. Furthermore, the following perturbation result is known.

Theorem 3.4. [11, XIII.4, Cor. 2] Let $T$ be a selfadjoint operator on $\mathcal{H}$ and $\checkmark$ relatively compact w.r.t. $T$. Then

(i) $H:=T+V$ is closed on $\operatorname{dom}(T)$ and

(ii) $\sigma_{e 5}(H)=\sigma_{e}(T)$.

From Theorems 3.3 and 3.4 we immediately conclude that for all $H \in \Omega_{2}$ the spectrum of $\mathcal{H}$ is of the form

$$
\sigma(H)=\sigma(T) \cup\left\{\lambda_{1}, \lambda_{2}, \ldots\right\},
$$

with isolated eigenvalues $\lambda_{i} \in \mathbb{C}$.

Strong resolvent convergence Let $P_{n}: \mathcal{H} \rightarrow \mathcal{H}_{n}$ be defined as in Sect. 2 and set $V_{n}:=\left.P_{n} V\right|_{\mathcal{H}_{n}}$.

Lemma 3.5. For $V_{n}$ defined as above, we have the following

(i) $\left(V_{n}\right)^{*}=\left(V^{*}\right)_{n}$ (i.e. compression to $\mathcal{H}_{n}$ commutes with taking the adjoint) and

(ii) $V_{n} P_{n} \rightarrow V$ strongly in $\mathcal{H}$.

(iii) $V_{n}^{*} P_{n} \rightarrow V^{*}$ strongly in $\mathcal{H}$.

Proof. Assertion (i) is easily shown by an analogous calculation to (2.7).

To see assertion (ii), let $u \in \mathcal{H}$ and note that then $P_{n} u \rightarrow u$ strongly. By continuity of $V$, it immediately follows that $V P_{n} u \rightarrow V u$ in $\mathcal{H}$. Hence, from the definition of $V_{n}$ we conclude that

$$
V_{n} P_{n} u=\left.P_{n} V\right|_{\mathcal{H}_{n}} P_{n} u=\underbrace{P_{n}}_{\rightarrow I \text { strongly }} \underbrace{V P_{n} u}_{\rightarrow V u} \rightarrow V u .
$$

Assertion (iii) now immediately follows by combining (i) and (ii).

The next lemma shows that even the perturbed operators $H_{n}$ converge in strong resolvent sense.

Lemma 3.6. For $H \in \Omega_{2}$ and $H_{n}=\left.P_{n} H\right|_{\mathcal{H}_{n}}$, one has $H_{n} \rightarrow H$ and $H_{n}^{*} \rightarrow$ $H^{*}$ in strong resolvent sense.

Proof. This follows from [4, Cor. 3.5], since

$$
\left\|(T-z)^{-1}\right\|^{-1},\left\|\left(T_{n}-z\right)^{-1}\right\|^{-1} \geq \operatorname{dist}(z, \mathbb{R}),
$$

which tends to $\infty$ as $z \rightarrow \mathrm{i} \infty$, and $\|V\|,\left\|V_{n}\right\|$ are uniformly bounded.

The algorithm The algorithm for $\Omega_{2}, \Lambda_{2}$ is defined analogously to that in Sect. 2. Namely, we define $G_{n}^{\mathbb{C}}:=\frac{1}{n}(\mathbb{Z}+\mathrm{i} \mathbb{Z}) \cap B_{n}(0) \subset \mathbb{C}$.

$$
\Gamma_{n}^{(2)}(H):=\left\{\lambda \in G_{n}^{\mathbb{C}} \mid \min \left\{s\left(H_{n}-\lambda\right), s\left(H_{n}^{*}-\bar{\lambda}\right)\right\} \leq \frac{1}{n}\right\} \cup \Gamma_{n}^{(1)}(T) .
$$


Note that we have $\min \left\{s(M-\lambda), s\left(M^{*}-\bar{\lambda}\right)\right\}=\left\|(M-\lambda)^{-1}\right\|^{-1}$ for any $n \times n$ matrix $M$ (cf. [9]). Since we have already shown that $\Gamma_{n}^{(1)}$ approximates $\sigma(T)$ correctly and that $\sigma(T)=\sigma_{e}(T)=\sigma_{e 5}(H)$, we know that $\Gamma_{n}^{(2)}$ will not miss anything in $\sigma_{e 5}(H)$. Thus, it only remains to prove absence of spectral pollution and spectral inclusion for the discrete set $\sigma(H) \backslash \sigma_{e 5}(H)$ for the algorithm

$$
\tilde{\Gamma}_{n}(H):=\left\{\lambda \in G_{n}^{\mathbb{C}} \mid \min \left\{s\left(H_{n}-\lambda\right), s\left(H_{n}^{*}-\bar{\lambda}\right)\right\} \leq \frac{1}{n}\right\}
$$

This will be done in the remainder of this section.

However, let us first take a moment to assure that $\Gamma_{n}^{(2)}$ defines a reasonable algorithm. Clearly, each $\Gamma_{n}^{(2)}$ depends only on the matrix elements $\left\langle T e_{i}^{(n)}, e_{j}^{(n)}\right\rangle$ and $\left\langle V e_{i}^{(n)}, e_{j}^{(n)}\right\rangle, 1 \leq i, j \leq k_{n}$. Moreover, by Lemma 2.4 it only requires finitely many algebraic operations on these numbers to determine whether $\lambda \in G_{n}^{\mathbb{C}}$ belongs to the set $\left\{\lambda \mid \min \left\{s\left(H_{n}-\lambda\right), s\left(H_{n}^{*}-\bar{\lambda}\right)\right\} \leq \frac{1}{n}\right\}$. Finally, since $\Lambda_{2}$ contains all matrix elements $\left\langle T e_{i}^{(n)}, e_{j}^{(n)}\right\rangle$, it follows from the comments made in Sect. 2 that $\Gamma_{n}^{(1)}$ is an admissible algorithm as well.

Remark 3.7. We note that the choice $\frac{1}{n}$ as an upper bound for $s\left(H_{n}-\lambda\right)$ in (3.1) is arbitrary. The proof below will show that one could equally well have chosen

$$
\Xi_{n}(H):=\left\{\lambda \in G_{n}^{\mathbb{C}} \mid \min \left\{s\left(H_{n}-\lambda\right), s\left(H_{n}^{*}-\bar{\lambda}\right)\right\} \leq \frac{3}{n}\right\} \cup \Gamma_{n}^{(1)}(T)
$$

instead of $\Gamma_{n}^{(2)}(H)$. This fact will be used in Sect. 4 .

Spectral pollution Let us prove that the approximation $\Gamma_{n}^{(2)}(H)$ does not have spectral pollution for $H \in \Omega_{2}$. To this end, note that again $\tilde{\Gamma}_{n}(H) \subset$ $\sigma_{\varepsilon}\left(H_{n}\right)$ for $\varepsilon>0$ fixed and $n$ large enough. According to [5, Th. 3.6 ii)], $\varepsilon$-pseudospectral pollution of the approximation $H_{n} \rightarrow H$ is confined to

$$
\sigma_{e}\left(\left(H_{n}\right)_{n \in \mathbb{N}}\right) \cup \sigma_{e}\left(\left(H_{n}^{*}\right)_{n \in \mathbb{N}}\right)^{*} \cup \bigcup_{\delta \in(0, \varepsilon]} \Lambda_{e, \delta}\left(\left(H_{n}\right)_{n \in \mathbb{N}}\right)
$$

Hence, for any sequence $\lambda_{n} \in \tilde{\Gamma}_{n}(H)$ with $\lambda_{n} \rightarrow \lambda \in \mathbb{C}$ we have

$$
\lambda \in \bigcap_{\varepsilon>0}\left(\sigma_{\varepsilon}(H) \cup \sigma_{e}\left(H_{n}\right)_{n \in \mathbb{N}} \cup \sigma_{e}\left(\left(H_{n}^{*}\right)_{n \in \mathbb{N}}\right)^{*} \cup \bigcup_{\delta \in(0, \varepsilon]} \Lambda_{e, \delta}\left(\left(H_{n}\right)_{n \in \mathbb{N}}\right)\right) .
$$

We conclude with the following

Lemma 3.8. It follows from (3.2) that

$$
\lambda \in \sigma(H) \cup \sigma_{e}\left(\left(H_{n}\right)_{n \in \mathbb{N}}\right) \cup \sigma_{e}\left(\left(H_{n}^{*}\right)_{n \in \mathbb{N}}\right)^{*} .
$$

Proof. Let (3.2) hold. Then

- Either there exists $\varepsilon_{0}>0$ such that $\lambda \in \sigma_{\varepsilon}(T) \cup \sigma_{e}\left(\left(H_{n}\right)_{n \in \mathbb{N}}\right) \cup \sigma_{e}$ $\left(\left(H_{n}^{*}\right)_{n \in \mathbb{N}}\right)^{*}$ for all $\varepsilon \in\left(0, \varepsilon_{0}\right)$, or 
- there exists a sequence $\varepsilon_{k}$ with $\varepsilon_{k} \searrow 0$ and $\lambda \in \bigcup_{\delta \in\left(0, \varepsilon_{k}\right]} \Lambda_{e, \delta}\left(\left(H_{n}\right)_{n \in \mathbb{N}}\right)$ for all $k$.

In the first case, it follows that

$$
\begin{aligned}
\lambda & \in \bigcap_{\varepsilon>0}\left(\sigma_{\varepsilon}(H) \cup \sigma_{e}\left(\left(H_{n}\right)_{n \in \mathbb{N}}\right) \cup \sigma_{e}\left(\left(H_{n}^{*}\right)_{n \in \mathbb{N}}\right)^{*}\right) \\
& =\left(\bigcap_{\varepsilon>0} \sigma_{\varepsilon}(H)\right) \cup \sigma_{e}\left(\left(H_{n}\right)_{n \in \mathbb{N}}\right) \cup \sigma_{e}\left(\left(H_{n}^{*}\right)_{n \in \mathbb{N}}\right)^{*} \\
& =\sigma(H) \cup \sigma_{e}\left(\left(H_{n}\right)_{n \in \mathbb{N}}\right) \cup \sigma_{e}\left(\left(H_{n}^{*}\right)_{n \in \mathbb{N}}\right)^{*} .
\end{aligned}
$$

In the second case, we have

$$
\begin{aligned}
\lambda & \in \bigcap_{k \in \mathbb{N}} \bigcup_{\delta \in\left(0, \varepsilon_{k}\right]} \Lambda_{e, \delta}\left(\left(H_{n}\right)_{n \in \mathbb{N}}\right) \\
& \subset \sigma_{e}\left(\left(H_{n}\right)_{n \in \mathbb{N}}\right)
\end{aligned}
$$

by Lemma 2.7 (ii). Next, by [3, Th. 6.1] we have $\sigma_{e}\left(\left(H_{n}\right)_{n \in \mathbb{N}}\right) \cup \sigma_{e}\left(\left(H_{n}^{*}\right)_{n \in \mathbb{N}}\right)^{*}$ $\subset W_{e}(H)$ and hence $\lambda \in \sigma(H) \cup W_{e}(H)$. In order to exclude spectral pollution it only remains to prove $W_{e}(H) \subset \sigma(H)$.

Lemma 3.9. For $H=T+V \in \Omega_{2}$ one has $W_{e}(H) \subset \sigma_{e}(H)$.

Proof. Let $H=T+V$ with $T$ selfadjoint, semibounded and $V \in L(\mathcal{H})$ such that $V, V^{*}$ are $T$-compact. Then denoting $\operatorname{Re}(V):=\frac{1}{2}\left(V+V^{*}\right)$ and $\operatorname{Im}(V):=$ $\frac{1}{2 i}\left(V-V^{*}\right)$ we have that $V=\operatorname{Re}(V)+\mathrm{i} \operatorname{Im}(V)$ with $\operatorname{Re}(V), \operatorname{Im}(V)$ relatively compact w.r.t. $T$. Applying [3, Th. 4.5] we conclude that $W_{e}(H)=W_{e}(T)$.

But now by our assumptions on $T$, we can see from [3, Th. 3.8] that

$$
W_{e}(T)=\operatorname{conv}\left(\widehat{\sigma}_{e}(T)\right) \backslash\{ \pm \infty\}=\sigma_{e}(T)=\sigma_{e}(H) .
$$

Note that the previous lemma is the only place in which we need the semiboundedness assumption in the definition of $\Omega_{2}$. Overall we have shown that for any sequence $\lambda_{n} \in \tilde{\Gamma}_{n}(H)$ which converges to some $\lambda \in \mathbb{C}$ we necessarily have $\lambda \in \sigma(H)$, in other words, spectral pollution does not exist.

Spectral inclusion It remains to show that the approximation $\left(\Gamma_{n}^{(2)}(H)\right)$ is spectrally inclusive, i.e. that for any $\lambda \in \sigma(H)$ there exists a sequence $\lambda_{n} \in$ $\Gamma_{n}^{(2)}(H)$ such that $\lambda_{n} \rightarrow \lambda$. As explained above, the existence of such a sequence is already guaranteed for all $\lambda \in \sigma_{e 5}(H)$.

Lemma 3.10. For every $\lambda \in \sigma(H) \backslash \sigma_{e 5}(H)$ there exists a sequence $\lambda_{n} \in \tilde{\Gamma}(H)$ with $\lambda_{n} \rightarrow \lambda$.

Proof. First note that by Theorem $3.3 \lambda$ is an isolated point. Moreover, we have seen in the proof of Lemma 3.9 that $\sigma_{e}\left(\left(H_{n}\right)_{n \in \mathbb{N}}\right) \cup \sigma_{e}\left(\left(H_{n}^{*}\right)_{n \in \mathbb{N}}\right)^{*} \subset$ $\sigma_{e}(H)$ and hence $\lambda$ does not belong to this set either. From Lemma 3.6 and [5, Th. $2.3 \mathrm{i})$ ] we conclude that there exists a sequence $\mu_{n} \in \sigma\left(H_{n}\right)$ with $\mu_{n} \rightarrow \lambda$.

Now, by definition of $G_{n}^{\mathbb{C}}$, for each $n$ there exists $\lambda_{n} \in G_{n}^{\mathbb{C}}$ such that $\left|\mu_{n}-\lambda_{n}\right|<\frac{1}{n}$ and hence $\left\|\left(H_{n}-\lambda_{n}\right)^{-1}\right\|_{L\left(\mathcal{H}_{n}\right)} \geq n$ which implies $\lambda_{n} \in \tilde{\Gamma}_{n}(H)$. Since $\left|\mu_{n}-\lambda_{n}\right| \rightarrow 0$ and $\mu_{n} \rightarrow \lambda$, it follows that $\lambda_{n} \rightarrow \lambda$. 
Conclusion Overall we have shown that

$\left(\mathrm{a}^{\prime}\right)$ If $\lambda_{n} \in \Gamma_{n}^{(2)}(H)$ and $\lambda_{n} \rightarrow \lambda$, then $\lambda \in \sigma(H)$.

$\left(\mathrm{b}^{\prime}\right)$ If $\lambda \in \sigma(H)$, then there exist $\lambda_{n} \in \Gamma_{n}^{(2)}(H)$ with $\lambda_{n} \rightarrow \lambda$.

By Proposition 2.8 this implies $d_{\mathrm{AW}}\left(\Gamma_{n}^{(2)}(H), \sigma(H)\right) \rightarrow 0$.

\section{Application to Schrödinger Operators}

In this section we will apply the results of Sects. 2 and 3 to Schrödinger operators on $L^{2}\left(\mathbb{R}^{d}\right)$. More specifically, fix a continuous, monotone decreasing function $g:[0, \infty) \rightarrow[0, \infty)$ with $g(t) \rightarrow 0$ as $t \rightarrow \infty$ and let $M>0$. We define

$$
\Omega_{3}:=\left\{-\Delta+V\left|V \in \mathcal{C}^{1}(\mathbb{R}, \mathbb{C}),\|\nabla V\|_{\infty} \leq M,\right| V(x) \mid \leq g(|x|)\right\} .
$$

By the above definition, every $H \in \Omega_{3}$ is a relatively compact perturbation of the free Laplacian with domain $H^{2}\left(\mathbb{R}^{d}\right)$ (cf. e.g. [10, Ch. V, Lemma 5.8]). In fact, our assumptions on $V$ have been chosen such that every $H \in \Omega_{3}$ even satisfies all conditions formulated in the set $\Omega_{2}$ in Theorem 3.1.

In order to define the computational problem, we choose a finite lattice in $\mathbb{R}^{d}$

$$
L_{n}:=\left\{\frac{i}{n}\left|i \in \mathbb{Z}^{d},\right| i \mid<n\right\} .
$$

Moreover, let $\mathcal{H}_{n}$ denote the subspace of $L^{2}\left(\mathbb{R}^{d}\right)$ spanned by all characteristic functions of cubes of edge length $\frac{1}{n}$ with centres inside a ball of radius $n$ :

$$
\widehat{\mathcal{H}}_{n}:=\operatorname{span}\left\{\chi_{i+\left[0, \frac{1}{n}\right)^{d}} \mid i \in L_{n}\right\}
$$

It is easily seen by smooth approximation that $P_{\widehat{\mathcal{H}}_{n}} \rightarrow I$ strongly in $L^{2}\left(\mathbb{R}^{d}\right)$. However, none of the basis functions $\chi_{i+\left[0, \frac{1}{n}\right)^{d}}$ are contained in the domain of $-\Delta$. In order to circumvent this, the space we will actually work with will be

$$
\mathcal{H}_{n}:=\operatorname{span}\left\{\widehat{\chi}_{i+\left[0, \frac{1}{n}\right)^{d}} \mid i \in L_{n}\right\}
$$

where the hat denotes the Fourier transform in $L^{2}\left(\mathbb{R}^{d}\right)$. For any enumeration $i_{k}$ of the set $L_{n}$, we define

$$
e_{k}^{(n)}:=n^{\frac{d}{2}} \cdot \widehat{\chi}_{i_{k}+\left[0, \frac{1}{n}\right)^{d}}
$$

where the normalisation constant $n^{\frac{d}{2}}$ is chosen such that $\left\|e_{k}^{(n)}\right\|_{L^{2}\left(\mathbb{R}^{d}\right)}=1$ for all $n \in \mathbb{N}$. These are smooth functions in $L^{2}\left(\mathbb{R}^{d}\right)$ and it is easily checked that their first and second derivatives are again in $L^{2}\left(\mathbb{R}^{d}\right)$.

Lemma 4.1. We have $P_{\mathcal{H}_{n}} \rightarrow I$ strongly in $L^{2}\left(\mathbb{R}^{d}\right)$ and for any $n \in \mathbb{N}$ the set $\left\{e_{k}^{(n)}\right\}_{k=1}^{\# L_{n}}$ form an orthonormal basis of $\mathcal{H}_{n}$. 
Proof. This follows immediately from the unitarity of the Fourier transform and the equality

$$
\begin{aligned}
& \left\|\sum_{k}\left\langle f, n^{\frac{d}{2}} \chi_{i_{k}+\left[0, \frac{1}{n}\right)^{d}}\right\rangle n^{\frac{d}{2}} \chi_{i_{k}+\left[0, \frac{1}{n}\right)^{d}}-f\right\|_{L^{2}\left(\mathbb{R}^{d}\right)} \\
& \quad=\left\|\sum_{k}\left\langle\hat{f}, e_{k}^{(n)}\right\rangle e_{k}^{(n)}-\hat{f}\right\|_{L^{2}\left(\mathbb{R}^{d}\right)}
\end{aligned}
$$

We note that the functions $e_{k}^{(n)}$ can be calculated explicitly. Indeed, one has

$$
e_{k}^{(n)}(\xi)=\left(\frac{n}{2 \pi}\right)^{\frac{d}{2}} \prod_{j=1}^{d} \frac{e^{\mathrm{i} \xi_{j}\left(\left(i_{k}\right)_{j}+\frac{1}{n}\right)}-e^{\mathrm{i} \xi_{j}\left(i_{k}\right)_{j}}}{\xi_{j}},
$$

where $\left(i_{k}\right)_{j}$ denotes the $j$ 'th component of the vector $i_{j}$ and $\xi=\left(\xi_{1}, \ldots, \xi_{d}\right) \in$ $\mathbb{R}^{d}$. Using this explicit representation, it can be easily seen that we have the following.

Lemma 4.2. For each $n \in \mathbb{N}$ one has

$$
\left\|e_{k}^{(n)}\right\|_{\infty},\left\|\nabla e_{k}^{(n)}\right\|_{\infty} \leq(2 \pi)^{-\frac{d}{2}} d n^{3-\frac{d}{2}}
$$

for all $k \in\{1, \ldots, n\}$.

Proof. From the definition of $e_{k}^{(n)}$ it follows by direct calculation that

$$
\begin{aligned}
&\left\|e_{k}^{(n)}\right\|_{\infty}<(2 \pi n)^{-\frac{d}{2}} \\
&\left\|\partial_{j} e_{k}^{(n)}\right\|_{\infty}<(2 \pi)^{-\frac{d}{2}} \frac{n^{-\frac{d}{2}+1}}{2}\left(\left(\left(i_{k}\right)_{j}+\frac{1}{n}\right)^{2}-\left(i_{k}\right)_{j}^{2}\right)
\end{aligned}
$$

from which the assertion follows. Note that the bound in the second equation can be made independent of $k$, because $i_{k} \in L_{n} \subset B_{n}(0)$ for all $k$.

The information accessible to the algorithm will be the set

$$
\Lambda_{3}:=\Lambda_{3}^{(1)} \cup \Lambda_{3}^{(2)} \cup \Lambda_{3}^{(3)} \cup \Lambda_{3}^{(4)},
$$

with

$$
\begin{aligned}
& \Lambda_{3}^{(1)}=\left\{\rho_{x} \mid x \in \mathbb{R}^{d}\right\} \\
& \Lambda_{3}^{(2)}=\left\{e_{k}^{(n)}(i) \mid i \in l^{-1} \mathbb{Z}^{d}, l \in \mathbb{N}, k \in\{1, \ldots, n\}, n \in \mathbb{N}\right\} \\
& \Lambda_{3}^{(3)}=\left\{\frac{n \delta_{m k}}{3} \sum_{j=1}^{d}\left(\left((i)_{j}+\frac{1}{n}\right)^{3}-(i)_{j}^{3}\right) \mid i \in L_{n}, m, k \in\left\{1, \ldots, \# L_{n}\right\}, n \in \mathbb{N}\right\} \\
& \Lambda_{3}^{(4)}=\left\{g\left(l \frac{1}{2 d}\right) \mid l \in \mathbb{N}\right\}
\end{aligned}
$$

where $\rho_{x}(V)=V(x)$ are the evaluation functionals and $e_{k}^{(n)}(i)$ denote constant functions that map $V$ to the number $e_{k}^{(n)}(i)$. The meaning of the constants $\frac{n \delta_{m k}}{3} \sum_{j=1}^{d}\left(\left((i)_{j}+\frac{1}{n}\right)^{3}-(i)_{j}^{3}\right)$ will become clear later on.

Together, $\Omega_{3}$ and $\Lambda_{3}$ define a computational problem $\left(\Omega_{3}, \Lambda_{3}, \sigma(\cdot)\right)$. The main result of this section is the following. 
Theorem 4.3. For $\Omega_{3}$ and $\Lambda_{3}$ defined as above, we have $\operatorname{SCI}\left(\Omega_{3}, \Lambda_{3}, \sigma(\cdot)\right)=$ 1.

The proof of Theorem 4.3 will be by reduction to Theorem 3.1. In order to accomplish this, we need to be able to compute the matrix elements $\left\langle(-\Delta+V) e_{i}, e_{j}\right\rangle$ by performing only a finite number of algebraic operations on a finite number of values of $V$. This will be the main difficulty.

\subsection{Proof of Theorem 4.3}

We first show that the spaces $\mathcal{H}_{n}$ defined in (4.2) are indeed a reasonable choice for the problem at hand. More precisely, we have

Lemma 4.4. The union $\bigcup_{n \in \mathbb{N}} \mathcal{H}_{n}$ is a core for $-\Delta$.

Proof. By means of the Fourier transform the assertion is equivalent to the space $\bigcup_{n \in \mathbb{N}} \widehat{\mathcal{H}}_{n}$ being a core for the multiplication operator $u \mapsto|\xi|^{2} u$ in $L^{2}\left(\mathbb{R}^{d}\right)$. To verify this, we have to show that for every $u \in \operatorname{dom}\left(\left|\xi^{2}\right|\right)$ there exists a sequence $u_{n} \in \mathcal{H}_{n}$ such that

(i) $\left\|u_{n}-u\right\|_{L^{2}\left(\mathbb{R}^{d}\right)} \rightarrow 0$,

(ii) $\left\||\xi|^{2}\left(u_{n}-u\right)\right\|_{L^{2}\left(\mathbb{R}^{d}\right)} \rightarrow 0$

Point (i) is easily shown by choosing

$$
u_{n}:=\sum_{i \in L_{n}}\left\langle u, n^{\frac{d}{2}} \chi_{i+\left[0, \frac{1}{n}\right)^{d}}\right\rangle n^{\frac{d}{2}} \chi_{i+\left[0, \frac{1}{n}\right)^{d}} .
$$

Indeed, for smooth $u$ the $L^{2}$-convergence of $u_{n}$ to $u$ is standard, while the general case follows by a density argument. We omit the technical details. To show point (ii), let $R>0$ and decompose the norm in (ii) as

$$
\left\||\xi|^{2}\left(u_{n}-u\right)\right\|_{L^{2}\left(\mathbb{R}^{d}\right)}^{2}=\left.\left.\int_{B_{R}}|| \xi\right|^{2}\left(u_{n}-u\right)\right|^{2} d \xi+\left.\left.\int_{\mathbb{R}^{d} \backslash B_{R}}|| \xi\right|^{2}\left(u_{n}-u\right)\right|^{2} d \xi
$$

where $B_{R}$ denotes the ball of radius $R$ centered at 0 . We first estimate the second term on the right hand side. To this end, we let $u_{n}$ be defined by (4.4) and employ the shorthand notation $\chi_{i}:=n^{\frac{d}{2}} \chi_{i+\left[0, \frac{1}{n}\right)^{d}}$. On the whole space we have

$$
\begin{aligned}
\left\||\xi|^{2} u_{n}\right\|_{L^{2}\left(\mathbb{R}^{d} \backslash B_{R}\right)}^{2} & =\left\||\xi|^{2} \sum_{i \in L_{n}}\left\langle u, \chi_{i}\right\rangle \chi_{i}\right\|_{L^{2}\left(\mathbb{R}^{d} \backslash B_{R}\right)}^{2} \\
& \leq \sum_{i \in L_{n} \backslash B_{R}}\left|\left\langle u, \chi_{i}\right\rangle\right|^{2}\left\||\xi|^{2} \chi_{i}\right\|_{L^{2}\left(\mathbb{R}^{d}\right)}^{2} \\
& \leq \sum_{i \in L_{n} \backslash B_{R}}\|u\|_{L^{2}\left(i+\left[0, \frac{1}{n}\right)^{d}\right)}^{2} \| n^{\frac{d}{2}|\xi|^{2} \|_{L^{2}\left(i+\left[0, \frac{1}{n}\right)^{d}\right)}^{2}}
\end{aligned}
$$

where we have used the fact that $\operatorname{supp}\left(\chi_{i}\right) \cap \operatorname{supp}\left(\chi_{j}\right)=\emptyset$ for $i \neq j$. The factor $\left\|n^{\frac{d}{2}}|\xi|^{2}\right\|_{L^{2}\left(i+\left[0, \frac{1}{n}\right)^{d}\right)}$ on the right hand side is clearly bounded 


\section{Frank Rösler}

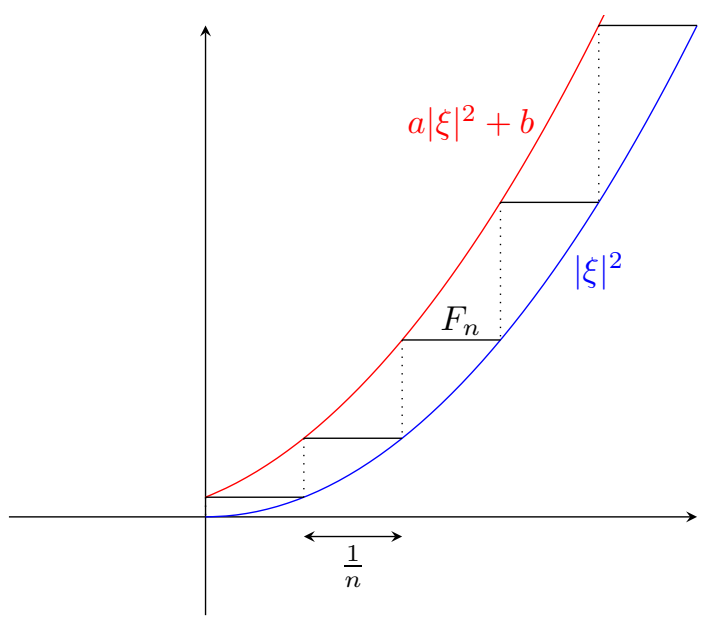

FiguRE 1. Sketch of function $F_{n}$

by $\sup _{\xi \in i+\left[0, \frac{1}{n}\right)^{d}}|\xi|^{2}$. Thus, if we define a function $F_{n}$ by

$$
F_{n}(\xi):=\sum_{i \in \frac{1}{n} \mathbb{Z}^{d}}\left(\sup _{\eta \in i+\left[0, \frac{1}{n}\right)^{d}}|\eta|^{2}\right) \chi_{i}
$$

then we will have (note that $F_{n}$ is constant on each of the cubes $i+\left[0, \frac{1}{n}\right)^{d}$ )

$$
\begin{aligned}
\left\||\xi|^{2} u_{n}\right\|_{L^{2}\left(\mathbb{R}^{d} \backslash B_{R}\right)}^{2} & \leq \sum_{i \in L_{n} \backslash B_{R}}\|u\|_{L^{2}\left(i+\left[0, \frac{1}{n}\right)^{d}\right)}^{2} F_{n}(\xi)^{2} \\
& =\sum_{i \in L_{n} \backslash B_{R}}\left\|F_{n}(\xi) u\right\|_{L^{2}\left(i+\left[0, \frac{1}{n}\right)^{d}\right)}^{2} \\
& \leq\left\|F_{n}(\xi) u\right\|_{L^{2}\left(\mathbb{R}^{d} \backslash B_{R-\frac{\sqrt{d}}{n}}^{2}\right)}
\end{aligned}
$$

Next, we note that it is easy to see that there exist constants $a, b>0$ such that $F_{n}(\xi) \leq a|\xi|^{2}+b$ uniformly in $n$ (see Fig. 1).

Overall we conclude that

$$
\begin{aligned}
\left\||\xi|^{2} u_{n}\right\|_{L^{2}\left(\mathbb{R}^{d} \backslash B_{R}\right)}^{2} & \leq\left\|F_{n}(\xi) u\right\|_{L^{2}\left(\mathbb{R}^{d} \backslash B_{R-\frac{\sqrt{d}}{n}}\right)}^{2} \\
& \leq\left\|\left(a|\xi|^{2}+b\right) u\right\|_{L^{2}\left(\mathbb{R}^{d} \backslash B_{R-1}\right)}^{2},
\end{aligned}
$$

where the last term on the right hand side is finite because by assumption $u \in \operatorname{dom}\left(|\xi|^{2}\right)$. In fact, from this last inequality we can see immediately that

$$
\left\||\xi|^{2} u_{n}\right\|_{L^{2}\left(\mathbb{R}^{d} \backslash B_{R}\right)}^{2} \rightarrow 0
$$


as $R \rightarrow \infty$ uniformly in $n$. Estimating the second term on the right hand side of Eq. (4.5) is now straightforward. We get

$$
\begin{aligned}
\left.\left.\int_{\mathbb{R}^{d} \backslash B_{R}}|| \xi\right|^{2}\left(u_{n}-u\right)\right|^{2} d \xi & \leq\left\||\xi|^{2} u_{n}\right\|_{L^{2}\left(\mathbb{R}^{d} \backslash B_{R}\right)}^{2}+\left\||\xi|^{2} u\right\|_{L^{2}\left(\mathbb{R}^{d} \backslash B_{R}\right)}^{2} \\
& \leq\left\|\left(a|\xi|^{2}+b\right) u\right\|_{L^{2}\left(\mathbb{R}^{d} \backslash B_{R-1}\right)}^{2}+\left\||\xi|^{2} u\right\|_{L^{2}\left(\mathbb{R}^{d} \backslash B_{R}\right)}^{2} .
\end{aligned}
$$

Now let $\varepsilon>0$ and choose $R$ so large that $\left\|\left(a|\xi|^{2}+b\right) u\right\|_{L^{2}\left(\mathbb{R}^{d} \backslash B_{R-1}\right)}^{2}+$ $\left\||\xi|^{2} u\right\|_{L^{2}\left(\mathbb{R}^{d} \backslash B_{R}\right)}^{2}<\varepsilon$. From Eq. (4.5) we then see that

$$
\begin{aligned}
\limsup _{n \rightarrow \infty}\left\||\xi|^{2}\left(u_{n}-u\right)\right\|_{L^{2}\left(\mathbb{R}^{d}\right)}^{2} & \leq\left.\left.\limsup _{n \rightarrow \infty} \int_{B_{R}}|| \xi\right|^{2}\left(u_{n}-u\right)\right|^{2} d \xi+\varepsilon \\
& \leq \limsup _{n \rightarrow \infty} R^{2} \int_{B_{R}}\left|u_{n}-u\right|^{2} d \xi+\varepsilon \\
& =\varepsilon
\end{aligned}
$$

because $u_{n} \rightarrow u$ in $L^{2}\left(\mathbb{R}^{d}\right)$. Since $\varepsilon$ was arbitrary, it follows that

$$
\limsup _{n \rightarrow \infty}\left\||\xi|^{2}\left(u_{n}-u\right)\right\|_{L^{2}\left(\mathbb{R}^{d}\right)}^{2}=0
$$

Our strategy for proving Theorem 4.3 is as follows. By the assumptions on $V$ stated in the definition of $\Omega_{3}$ and Lemma 4.4 we know that we have $\Omega_{3} \subset \Omega_{2}$, if we choose $\mathcal{H}=L^{2}\left(\mathbb{R}^{d}\right)$ and $\mathcal{H}_{n}$ as in (4.2). Hence, we already know from Theorem 3.1 that $\Gamma_{n}^{(2)}(H) \rightarrow \sigma(H)$ for all $H \in \Omega_{3}$. However, $\Gamma_{n}^{(2)}$ uses the matrix elements $\left\langle H e_{k}^{(n)}, e_{j}^{(n)}\right\rangle$, which we are not allowed to access in Theorem 4.3. Therefore, we will define a new algorithm $\Gamma_{n}^{(3)}$ which only accesses the information provided in $\Lambda_{3}$ and which satisfies $\Gamma_{n}^{(3)}(H) \approx$ $\Gamma_{n}^{(2)}(H)$ for $H \in \Omega_{3}$ in an appropriate sense.

The algorithm As described above, we need to approximate the matrix elements $\left\langle-\Delta e_{k}^{(n)}, e_{m}^{(n)}\right\rangle$ and $\left\langle V e_{k}^{(n)}, e_{m}^{(n)}\right\rangle$ using only a finite amount of information provided in the set $\Lambda_{3}$. We start with the Laplacian, which is the simpler case. Indeed, we have

$$
\begin{aligned}
\left\langle-\Delta e_{k}^{(n)}, e_{m}^{(n)}\right\rangle & =\left\langle|\xi|^{2} n^{\frac{d}{2}} \chi_{i_{k}+\left[0, \frac{1}{n}\right)^{d}}, n^{\frac{d}{2}} \chi_{i_{m}+\left[0, \frac{1}{n}\right)^{d}}\right\rangle \\
& =n^{d} \delta_{m k} \int_{i_{k}+\left[0, \frac{1}{n}\right)^{d}}|\xi|^{2} d \xi \\
& =\frac{n \delta_{m k}}{3} \sum_{j=1}^{d}\left(\left(\left(i_{k}\right)_{j}+\frac{1}{n}\right)^{3}-\left(i_{k}\right)_{j}^{3}\right) .
\end{aligned}
$$

Note that these are precisely the terms in the third factor in Eq. (4.3).

Next, we will compute the matrix elements $\left\langle V e_{k}^{(n)}, e_{m}^{(n)}\right\rangle$. Since any algorithm can only use finitely many values of $V$, we will have to perform an 
approximation procedure. To this end, let $l \in \mathbb{N}$ and define a lattice $P_{l} \subset \mathbb{R}^{d}$ by

$$
P_{l}:=\frac{1}{l} \mathbb{Z}^{d} \cap Q_{l},
$$

where $Q_{l}$ denotes the cube of edge length $l^{\frac{1}{2 d}}$ centered at 0 . Next, let

$$
V_{l}(x):=\sum_{i \in P_{l}} V(i) \chi_{i+\left[0, \frac{1}{l}\right)^{d}} .
$$

Lemma 4.5. For any function $f \in C^{1}\left(\mathbb{R}^{d}\right)$ one has

$$
\left\|f-\sum_{i \in P_{l}} f(i) \chi_{i+\left[0, \frac{1}{l}\right)^{d}}\right\|_{L^{\infty}\left(Q_{l}\right)} \leq \frac{\|\nabla f\|_{\infty}}{l},
$$

Proof. This follows immediately from the identity

$$
f(x)-f(i)=\int_{[i, x]} \nabla f(t) \cdot d t
$$

where $i \in P_{l}$ and $[i, x]$ denotes a line segment connecting $i$ to $x \in i+\left[0, \frac{1}{l}\right)^{d}$.

In order to define our approximation of $\left\langle V e_{k}^{(n)}, e_{m}^{(n)}\right\rangle$, we additionally introduce the step function approximation

$$
E_{k, l}(x):=\sum_{i \in P_{l}} e_{k}^{(n)}(l) \chi_{i+\left[0, \frac{1}{l}\right)^{d}} .
$$

Lemma 4.6. For $-\Delta+V \in \Omega_{3}$ one has

$$
\left|\left\langle V e_{k}^{(n)}, e_{m}^{(n)}\right\rangle-\left\langle V_{l} E_{k, l}, E_{m, l}\right\rangle\right| \leq \frac{3}{l^{\frac{1}{2}}}(M+g(0))(2 \pi)^{-\frac{d}{2}} n^{3-\frac{d}{2}} d+g\left(l^{\frac{1}{2 d}}\right),
$$

for all $l \in \mathbb{N}$, where $M$ is as in Eq. (4.1).

Proof. We calculate the error

$$
\begin{aligned}
& \left|\left\langle V e_{k}^{(n)}, e_{m}^{(n)}\right\rangle-\left\langle V_{l} E_{k, l}, E_{m, l}\right\rangle\right| \\
& \quad=\left|\sum_{i \in P_{l}} \int_{i+\left[0, \frac{1}{l}\right)} V e_{k}^{(n)} e_{m}^{(n)} d x-\sum_{i \in P_{l}} \int_{i+\left[0, \frac{1}{l}\right)} V_{l} E_{k, l} E_{m, l} d x+\int_{\mathbb{R}^{d} \backslash Q_{l}} V e_{k}^{(n)} e_{m}^{(n)} d x\right| \\
& \quad \leq \sum_{i \in P_{l}} \int_{i+\left[0, \frac{1}{l}\right)}\left|V e_{k}^{(n)} e_{m}^{(n)}-V_{l} E_{k, l} E_{m, l}\right| d x+\int_{\mathbb{R}^{d} \backslash Q_{l}}\left|V e_{k}^{(n)} e_{m}^{(n)}\right| d x \\
& \quad \leq \sum_{i \in P_{l}} \int_{i+\left[0, \frac{1}{l}\right)} l^{-1}\left\|\nabla\left(V e_{k}^{(n)} e_{m}^{(n)}\right)\right\|_{\infty} d x+g\left(l^{\frac{1}{2 d}}\right) \int_{\mathbb{R}^{d} \backslash Q_{l}}\left|e_{k}^{(n)} e_{m}^{(n)}\right| d x \\
& \quad=l^{-1}\left|Q_{l}\right|\left\|\nabla\left(V e_{k}^{(n)} e_{m}^{(n)}\right)\right\|_{\infty}+g\left(l^{\frac{1}{2 d}}\right)\left\|e_{k}^{(n)}\right\|_{L^{2}\left(\mathbb{R}^{d}\right)}\left\|e_{m}^{(n)}\right\|_{L^{2}\left(\mathbb{R}^{d}\right)} \\
& \leq l^{-1} l^{\frac{1}{2}}\left(\left\|\nabla V e_{k}^{(n)} e_{m}^{(n)}\right\|_{\infty}+\left\|V \nabla e_{k}^{(n)} e_{m}^{(n)}\right\|_{\infty}+\left\|V e_{k}^{(n)} \nabla e_{m}^{(n)}\right\|_{\infty}\right)+g\left(l^{\frac{1}{2 d}}\right) \\
& \leq \frac{3}{l^{\frac{1}{2}}}(M+g(0))(2 \pi)^{-\frac{d}{2}} n^{3-\frac{d}{2}} d+g\left(l^{\frac{1}{2 d}}\right),
\end{aligned}
$$

where we have used Lemma 4.5 in the third line and Lemma 4.2 and the fact that $\|V\|_{\mathcal{C}^{1}} \leq M$ in the last line. 
Corollary 4.7. If we denote $H_{n}:=\left.P_{n}(-\Delta+V)\right|_{\mathcal{H}_{n}}$ and $H_{n}^{l}:=\left.P_{n}(-\Delta)\right|_{\mathcal{H}_{n}}+$ $W^{l}$, where $W^{l}$ denotes the operator on $\mathcal{H}_{n}$ defined by the $n \times n$ matrix with elements $\left(W^{l}\right)_{k m}=\left\langle V_{l} E_{k, l}, E_{m, l}\right\rangle$ in the basis $\left\{e_{k}^{(n)}\right\}_{k=1}^{\# L_{n}}$, then

$$
\left\|H_{n}-H_{n}^{l}\right\|_{L\left(\mathcal{H}_{n}\right)} \leq \frac{3 d(M+g(0))\left(2 \pi n^{4}\right)^{-\frac{d}{2}}}{l^{\frac{1}{2}}}+n g\left(l^{\frac{1}{2 d}}\right) .
$$

Proof. By Lemma 4.6 the matrix elements of $H_{n}$ and $H_{n}^{l}$ satisfy $\mid\left(H_{n}\right)_{k m}-$ $\left(H_{n}^{l}\right)_{k m} \mid \leq \frac{3}{l^{\frac{1}{2}}}(M+g(0))(2 \pi)^{-\frac{d}{2}} n^{3-\frac{d}{2}} d+g\left(l^{\frac{1}{2 d}}\right)$. Now note that for any two matrices $A=\left(A_{k m}\right)$ and $B=\left(B_{k m}\right)$ one has

$$
\begin{aligned}
\|(A-B) x\|_{\mathcal{H}_{n}}^{2} & =\sum_{k=1}^{n}\left|\sum_{m=1}^{n}\left(A_{k m}-B_{k m}\right) x_{m}\right|^{2} \\
& \leq\left(\sup _{k, m}\left|A_{k m}-B_{k m}\right|\right) \sum_{k, m=1}^{n}\left|x_{m}\right|^{2} \\
& =n\left(\sup _{k, m}\left|A_{k m}-B_{k m}\right|\right)\|x\|_{\mathcal{H}_{n}}^{2} .
\end{aligned}
$$

This immediately implies the assertion.

We are finally ready to define our algorithm. Let $n \in \mathbb{N}$ and choose $l(n) \in \mathbb{N}$ large enough such that $\frac{3}{l(n)^{\frac{1}{2}}}(M+g(0))(2 \pi)^{-\frac{d}{2}} n^{3-\frac{d}{2}} d+g\left(l(n)^{\frac{1}{2 d}}\right)<$ $\frac{1}{2 n}$ (note that this can be done by a computer in finite time). Define for $H=-\Delta+V \in \Omega_{3}$

where

$$
\Lambda_{\Gamma_{n}^{(3)}}(H):=\Lambda_{\Gamma_{n}^{(3)}}^{(1)} \cup \Lambda_{\Gamma_{n}^{(3)}}^{(2)} \cup \Lambda_{\Gamma_{n}^{(3)}}^{(3)} \cup \Lambda_{\Gamma_{n}^{(3)}}^{(4)}
$$

$$
\begin{aligned}
& \Lambda_{\Gamma_{n}^{(3)}}^{(1)}=\left\{\rho_{i} \mid i \in P_{l(n)}\right\} \\
& \Lambda_{\Gamma_{n}^{(3)}}^{(2)}=\left\{e_{k}^{(n)}(i) \mid i \in P_{l(n)}, k \in\{1, \ldots, n\}\right\} \\
& \Lambda_{\Gamma_{n}^{(3)}}^{(3)}=\left\{\frac{n \delta_{m k}}{3} \sum_{j=1}^{d}\left(\left((i)_{j}+\frac{1}{n}\right)^{3}-(i)_{j}^{3}\right) \mid i \in L_{n}, m, k \in\left\{1, \ldots, \# L_{n}\right\}\right\} \\
& \Lambda_{\Gamma_{n}^{(3)}}^{(4)}=\left\{g\left(l^{\frac{1}{2 d}}\right) \mid l=0 \ldots l(n)\right\}
\end{aligned}
$$

and let

$$
\Gamma_{n}^{(3)}(H):=\left\{\lambda \in G_{n}^{\mathbb{C}} \mid\left\|\left(H_{n}^{l(n)}-\lambda\right)^{-1}\right\|^{-1} \leq \frac{2}{n}\right\} \cup \Gamma_{n}^{(1)}(-\Delta)
$$

with the convention that $\left\|\left(H_{n}^{l(n)}-\lambda\right)^{-1}\right\|^{-1}=0$ when $\lambda \in \sigma\left(H_{n}^{l(n)}\right)$. Note that $\Lambda_{\Gamma_{n}^{(3)}}(H)$ is a finite set for each $H \in \Omega_{3}$ and by Lemma 2.4 determining whether $\left\|\left(H_{n}^{l(n)}-\lambda\right)^{-1}\right\|^{-1} \leq \frac{2}{n}$ requires only finitely many algebraic operations on the matrix elements of $H_{n}^{l(n)}$ (which are contained in $\Lambda_{\Gamma_{n}^{(3)}}(H)$ ). Moreover, since $\Lambda_{3}$ contains all matrix elements of the Laplacian, we conclude that computing $\Gamma_{n}^{(1)}(-\Delta)$ can also be done by performing a finite amount of 
algebraic operations on the information provided. Overall, we conclude that each $\Gamma_{n}^{(3)}$ is an arithmetic algorithm in the sense of Definition 1.2.

Convergence It remains to prove that $\Gamma_{n}^{(3)}(H) \rightarrow \sigma(H)$ in the Attouch-Wets metric. To this end, let $\lambda \in G_{n}^{\mathbb{C}}$ and note that by the second resolvent identity we have

$$
\left(H_{n}^{l(n)}-\lambda\right)^{-1}-\left(H_{n}-\lambda\right)^{-1}=\left(H_{n}^{l(n)}-\lambda\right)^{-1}\left(H_{n}-H_{n}^{l(n)}\right)\left(H_{n}-\lambda\right)^{-1} .
$$

From (4.6) we conclude that

$$
\left|\left\|\left(H_{n}^{l(n)}-\lambda\right)^{-1}\right\|^{-1}-\left\|\left(H_{n}-\lambda\right)^{-1}\right\|^{-1}\right| \leq\left\|H_{n}-H_{n}^{l(n)}\right\| .
$$

Indeed if $\lambda \in \rho\left(H_{n}\right) \cap \rho\left(H_{n}^{l(n)}\right)$, this just follows by taking norms on both sides and using the reverse triangle inequality, while for $\lambda \in \sigma\left(H_{n}\right) \cup \sigma\left(H_{n}^{l(n)}\right)$ it can be seen by the following argument. W.l.o.g. assume that $\lambda \in \sigma\left(H_{n}\right) \backslash \sigma\left(H_{n}^{l(n)}\right)$. Then $\left\|\left(H_{n}-\lambda\right)^{-1}\right\|^{-1}=0$ and $\left\|\left(H_{n}^{l(n)}-\lambda\right)^{-1}\right\|^{-1}>0$. Assume for contradiction that (4.7) is false, i.e.

$$
\left\|H_{n}-H_{n}^{l(n)}\right\|<\left\|\left(H_{n}^{l(n)}-\lambda\right)^{-1}\right\|^{-1} .
$$

Then by a standard Neumann series argument (cf. [10, Sec. I.4.4]) it follows that $H_{n}$ is boundedly invertible, which contradicts the assumption $\lambda \in$ $\sigma\left(H_{n}\right)$. This argument is obviously symmetric in $H_{n}$ and $H_{n}^{l(n)}$.

Going back to (4.7) and recalling our specific choice of $l(n)$, we conclude that for all $\lambda \in G_{n}^{\mathbb{C}}$ one has

$$
\begin{aligned}
\|\|\left(H_{n}^{l(n)}-\lambda\right)^{-1}\left\|^{-1}-\right\|\left(H_{n}-\lambda\right)^{-1} & \|^{-1} \mid \\
& \leq\left\|H_{n}-H_{n}^{l(n)}\right\| \\
& \leq \frac{3 d(M+g(0))\left(2 \pi n^{4}\right)^{-\frac{d}{2}}}{l(n)^{\frac{1}{2}}}+n g\left(l(n)^{\frac{1}{2 d}}\right) \\
& \leq \frac{1}{2 n}
\end{aligned}
$$

Now, if $\lambda \in \Gamma_{n}^{(3)}(H)$ the above inequality implies that

$$
\begin{aligned}
\left\|\left(H_{n}-\lambda\right)^{-1}\right\|^{-1} & \leq\left\|\left(H_{n}^{l(n)}-\lambda\right)^{-1}\right\|^{-1}+\frac{1}{2 n} \\
& \leq \frac{2}{n}+\frac{1}{2 n} \\
& \leq \frac{3}{n}
\end{aligned}
$$


and hence $\lambda \in \Xi_{n}(H)$ (cf. Remark 3.7). Similarly, if $\lambda \in \Gamma_{n}^{(2)}(H)$ then

$$
\begin{aligned}
\left\|\left(H_{n}^{l(n)}-\lambda\right)^{-1}\right\|^{-1} & \leq\left\|\left(H_{n}-\lambda\right)^{-1}\right\|^{-1}+\frac{1}{n} \\
& \leq \frac{1}{n}+\frac{1}{2 n} \\
& \leq \frac{2}{n}
\end{aligned}
$$

and hence $\lambda \in \Gamma_{n}^{(3)}(H)$. Thus, we have the inclusions

$$
\Gamma_{n}^{(2)}(H) \subset \Gamma_{n}^{(3)}(H) \subset \Xi_{n}(H) .
$$

Since $\Gamma_{n}^{(2)}(H) \rightarrow \sigma(H)$ and $\Xi_{n}(H) \rightarrow \sigma(H)$ by Theorem 3.1 and Remark 3.7, we conclude that $\Gamma_{n}^{(3)}(H) \rightarrow \sigma(H)$ as well. This completes the proof of Theorem 4.3 .

\section{Acknowledgements}

The author would like to thank J. Ben-Artzi, A. Hansen and M. Marletta for helpful and inspiring discussions. Moreover, I would like to thank the anonymous reviewer for their helpful comments and corrections. This work was supported by the Engineering and Physical Sciences Research Council (UK): Grant EP/N020154/1 "QUEST: Quantitative Estimates in Spectral Theory and Their Complexity".

Open Access. This article is distributed under the terms of the Creative Commons Attribution 4.0 International License (http://creativecommons.org/licenses/by/4. $0 /$, which permits unrestricted use, distribution, and reproduction in any medium, provided you give appropriate credit to the original author(s) and the source, provide a link to the Creative Commons license, and indicate if changes were made.

Publisher's Note Springer Nature remains neutral with regard to jurisdictional claims in published maps and institutional affiliations.

\section{References}

[1] Boulton, L., Boussaid, N., Lewin, M.: Generalised Weyl theorems and spectral pollution in the Galerkin method. J. Spectr. Theory 2(4), 329-354 (2012)

[2] Ben-Artzi, J., Hansen, A.C., Nevanlinna, O., Seidel, M.: Can everything be computed? On the solvability complexity index and towers of algorithms. CoRR, arXiv:1508.03280 (2015)

[3] Bögli, S., Marletta, M., Tretter, C.: The essential numerical range for unbounded operators. arXiv preprint arXiv:1907.09599 (2019)

[4] Bögli, S.: Convergence of sequences of linear operators and their spectra. Integral Equ. Oper. Theory 88(4), 559-599 (2017)

[5] Bögli, S.: Local convergence of spectra and pseudospectra. J. Spectr. Theory 8(3), 1051-1098 (2018)

[6] Bögli, S., Siegl, P.: Remarks on the convergence of pseudospectra. Integr. Equ. Oper. Theory 80(3), 303-321 (2014) 
[7] Doyle, P., McMullen, C.: Solving the quintic by iteration. Acta Math. 163(3-4), 151-180 (1989)

[8] Edmunds, D.E., Evans, W.D.: Spectral Theory and Differential Operators (Oxford Mathematical Monographs). Oxford University Press, Oxford (1987)

[9] Hansen, A.C.: On the solvability complexity index, the $n$-pseudospectrum and approximations of spectra of operators. J. Am. Math. Soc. 24(1), 81-124 (2011)

[10] Kato, T.: Perturbation Theory for Linear Operators (Classics in Mathematics), reprint of the corr 2nd edn. Springer, Berlin (1995). reprint of the corr

[11] Reed, M., Simon, B.: Methods of Modern Mathematical Physics I: Functional Analysis. Academic Press Inc., New York (1980)

[12] Stampfli, J.G., Williams, J.P.: Growth conditions and the numerical range in a Banach algebra. Tôhoku Math. J. (2) 20, 417-424 (1968)

[13] Weidmann, J.: Lineare Operatoren in Hilberträumen. Teil 1. Mathematische Leitfäden. [Mathematical Textbooks]. B. G. Teubner, Stuttgart, 2000. Grundlagen. [Foundations]

Frank Rösler $(\bowtie)$

School of Mathematics

Cardiff University

Senghennydd Road

Cardiff CF24 4AG

UK

e-mail: RoslerF@cardiff.ac.uk

Received: March 14, 2019.

Revised: September 13, 2019. 